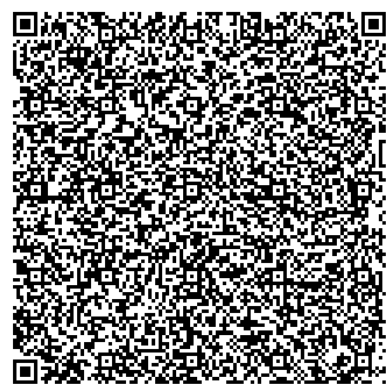

\title{
The Impact of Marketing Innovation on Customer Satisfaction in Aqaba Special Economic Zone Authority
}

\author{
Shaker Habis Nawafleh \\ AL Hussein Bin Talal University, Jordan \\ Prof. Dr. Suleiman Al-Khattab \\ AL Hussein Bin Talal University, Jordan
}

\begin{abstract}
:
The aim of this study was to explore the effect of marketing innovation on customer satisfaction in Aqaba Special Economic Zone Authority. Also, the study involved the effect of each one of the elements of marketing innovation (innovation in marketing, innovation in performance, innovation in culture and innovation in product) on the customer satisfaction level. A special questioner was developed to collect the data from (110) person, valid questioner was (102), which considered to be (92.7\%) of the participants. The result of analysis of the data by using SPSS 21 program; indicated that there was a statically significance strong positive relationship between customer satisfaction and marketing innovation, so (41\%) of the customer satisfaction might be explained by marketing innovation. And the strongest significance relationship in relation to the elements of marketing innovation was the relationship between innovation in performance and the customer satisfaction, while the innovation of marketing was in the second degree.The current study recommend to the Aqaba Special Economic Zone Authority to enhance the innovation of culture in between the employees to improve their customer satisfaction levels.

Keywords:

Aqaba Special Economic Zone Authority, Customer Satisfaction, Marketing Innovation, Innovation in Marketing, Innovation in Performance, Innovation in Culture and Innovation in Product

Citation

Nawafleh, Shaker Habis; Al-Khattab, Prof. Dr. Suleiman (2019); The Impact of Marketing Innovation on Customer Satisfaction in Aqaba Special Economic Zone Authority; Journal of Social Sciences (COES\&RJ-JSS), Vol.8, No.3, pp:399-417; https://doi.org/10.25255/jss.2019.8.3.399.417.
\end{abstract}

This work is licensed under a Creative Commons Attribution 4.0 International License. 
يو اجه العالم تحو لات هامة وتطور ات سريعة على جميع الأصعدة التقنية، السياسية، الاجتماعية والثقافية، و الاقتصادية

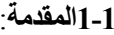

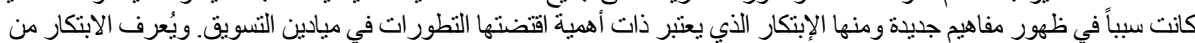

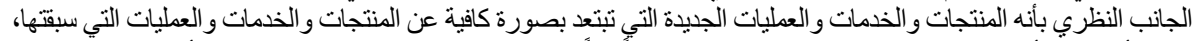

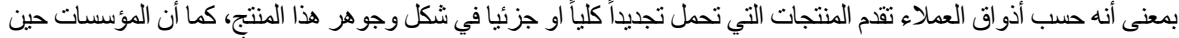

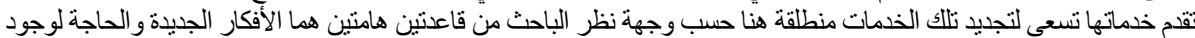

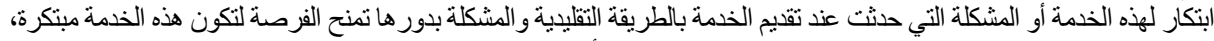

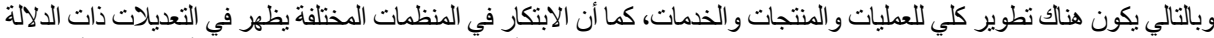

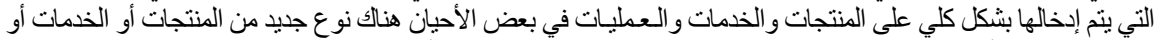

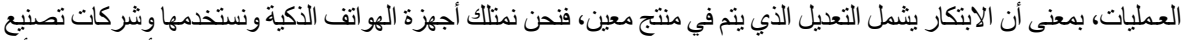

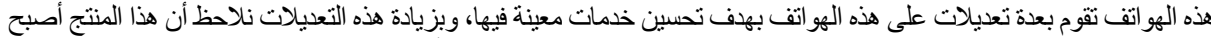

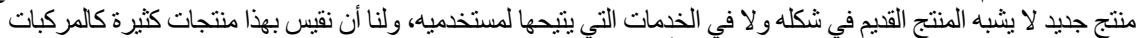

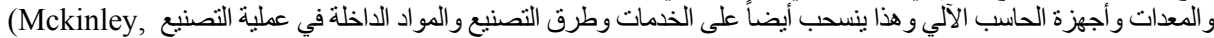

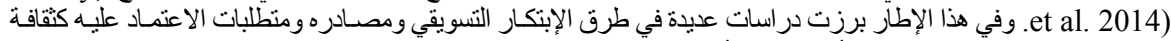

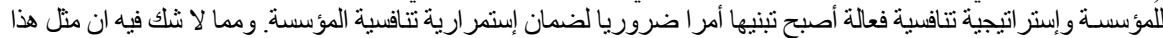

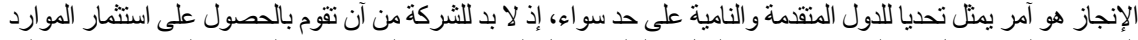

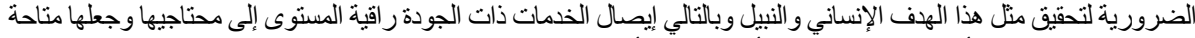

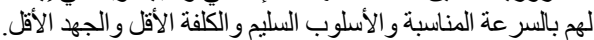

1-2 1-2 مشكلة الدراسة وأسئلتها:

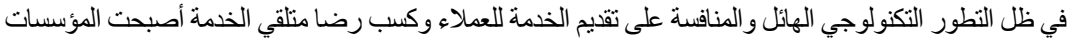

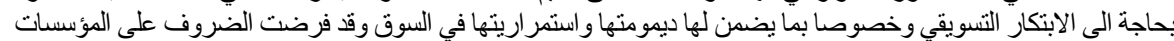

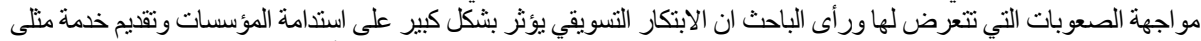

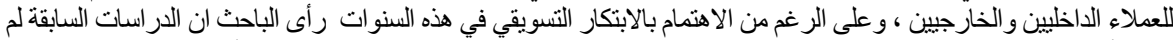

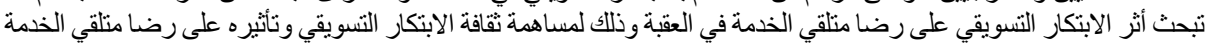

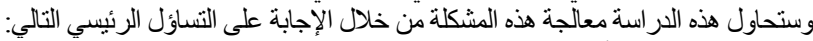
السؤال الرئيسي: ما أثر الابتكار التسويقي على رضا متلفي الخدمة في سلطة منطقة العقبة الاقتصادية الخاصة ؟ ويتفرع منه

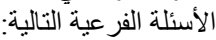

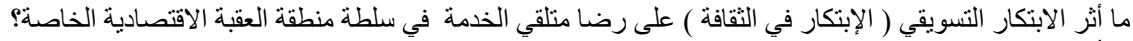

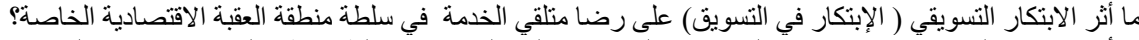

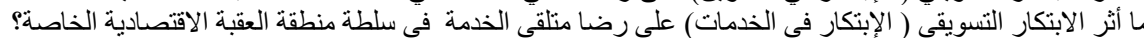

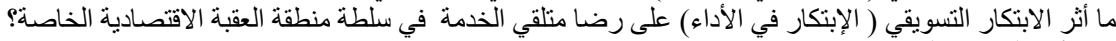

إن الابتكار هو المحرك الرئيسي للنمو في اقتصاد السوق، والقدرة على الابتكار امر بالغ الأهمية ليس فقط من

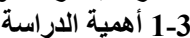

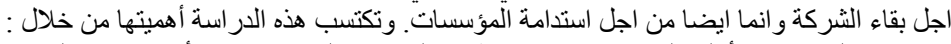
تعتبر هذه الدر اسة من أولى الدراسات ـ حسب اطلاع الباحث ـ التي تبحث عن أثر الإبتكار التسويقي على رضات رنا متلقي الخدمة في سلطة منطقة العقبة.

تظرّرها لموضو ع حيوي و هام و هو الإبتكار الذي يساعد المؤسسات على استقرار عملها والسبطرة على أنشطتها والمحافظة على زبائنها.

ثُبرز هذه الدر اسة أهمية طبيعة التوجه نحو الابتكار التسويقي كركيزة أساسية تعتمد عليها المؤسسة من اجل البقاء في القمة

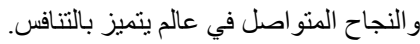

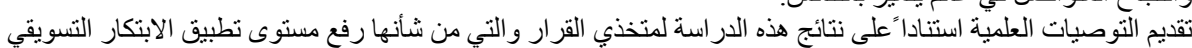
وكسب رضا متلقي الخدمة.

يأمل الباحث أن تكون هذه الدر اسة بمثابة بوصلة توجيهية للمسؤولين في المؤسسات للتركيز على الإبتكار بشكل عام و الابتكار

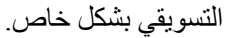

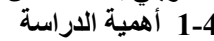

ان اختيار الباحث لموضو ع اثر الابتكار التسويقي على رضا متلقي الخدمة في سلطة منطقة العقبة على اعتبار

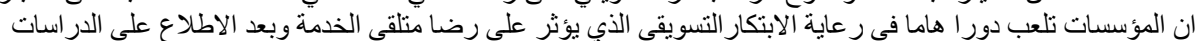
السابقة تسعى هذه الدر اسة الى تحقيق الأهداف التالية التالية:

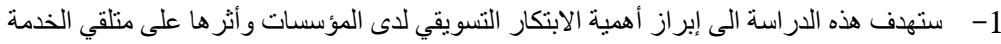

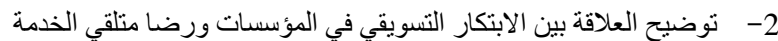
3- التعرف على أثر الابتكار التسويقي على رضا متلقي الخدمة في سلطة منطقة العقبة الاقتصادية الخاصة 
The Impact of Marketing Innovation on Customer Satisfaction ....

4- التعرف على أثر عناصر الابتكار التسويقي المتعددة على رضا متلقي الخدمة في سلطة منطقة العقبة الاقتصادية الخاصة.

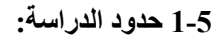

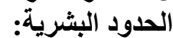

متلقي الخدمة في سلطة منطقة العقبة الاقتصادية الخاصة.

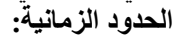

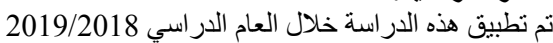

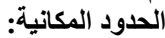

سلطة منطقة العقبة الاقتصادية الخاصة الإنة

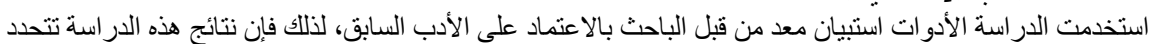

بدرجة صدق وثبات هذا الاستبيان.

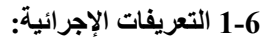

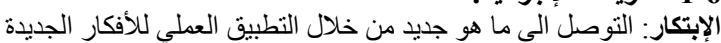

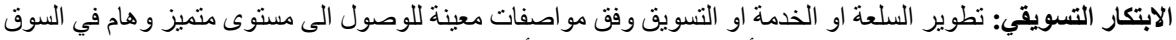

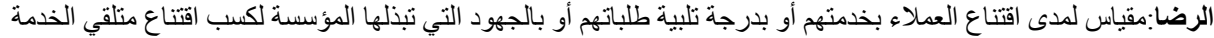

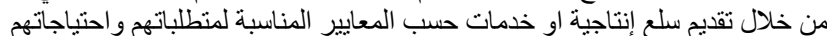

سلطة منطقة العقبة الاقتصادية الخاصة: هي مؤسسة حكومية مستقلة ماليا و اداريا انشأت بأمر ملكي في عام 2001 لتكون

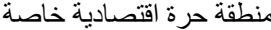

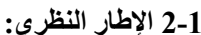

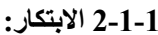

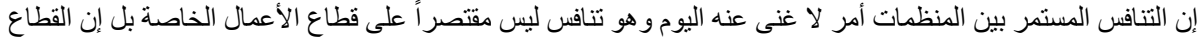

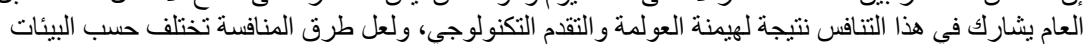

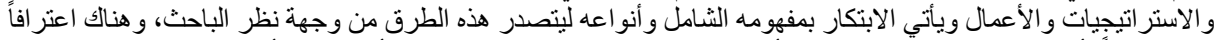
يتز ايد يومياً بأهمية الابتكار للمنظمات لتكون أكثر ديناميكية وهو يمثل عملية التجديد الأساسية في أي منظمة (رفاعي،

(2012

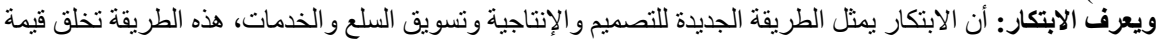

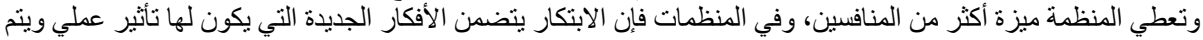

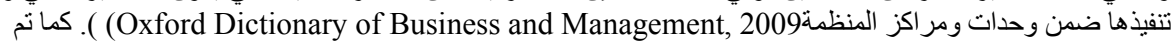

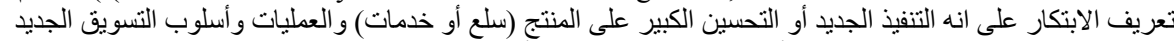

و والطرق التنظيمية الجديدة في ممارسة الأعمال وتنظيم مكان العمل أو العلاقلاقات الخارجية (Tiwari, 2008).

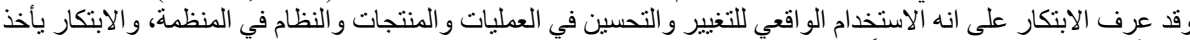

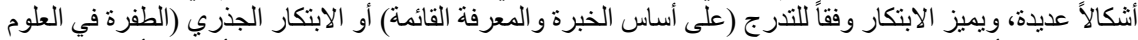

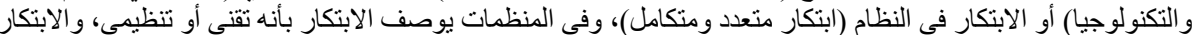

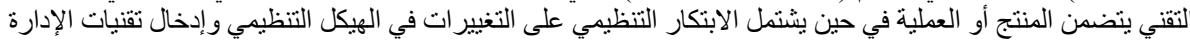

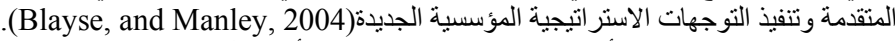

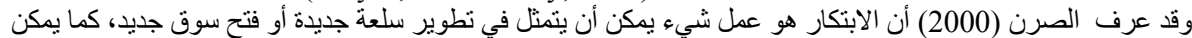

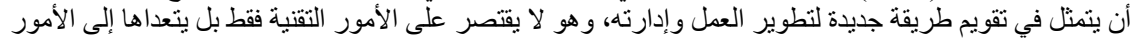

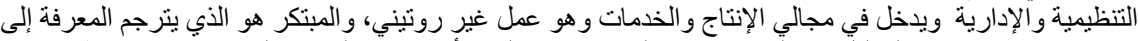

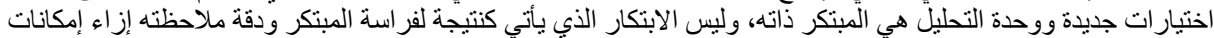

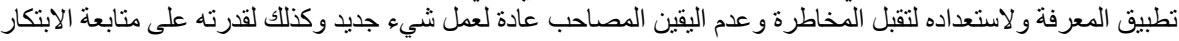

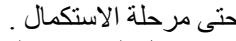

ومن خلال التعريفات السابقة يمكن الاستتتاج و القول بأن الابتكار هو التطبيق العملي للإختر اع او عملية صنع سلعة جديدة او

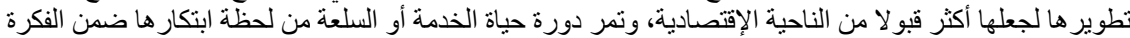

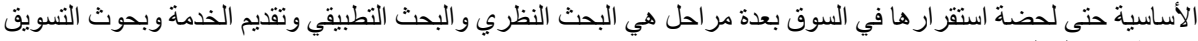

و الانطلاق و العملية الإنتاجية.

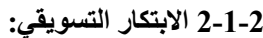

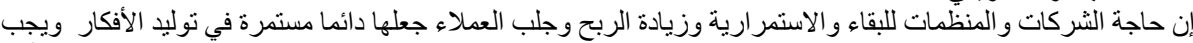

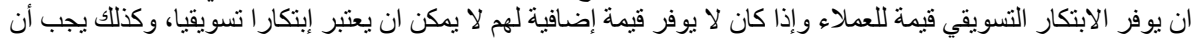

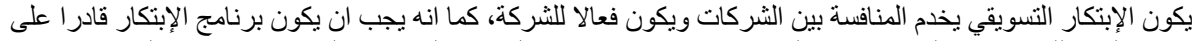

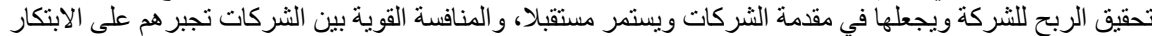

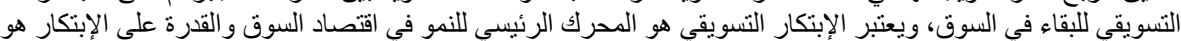

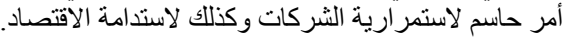




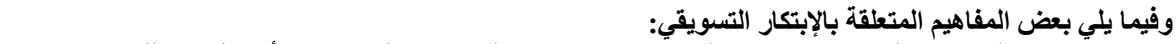

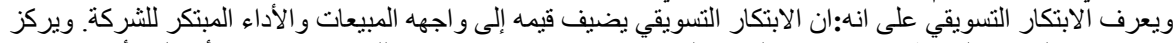

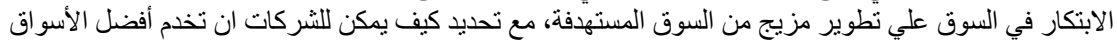

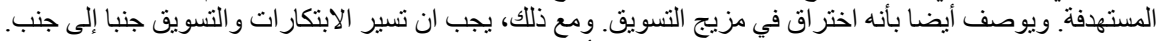

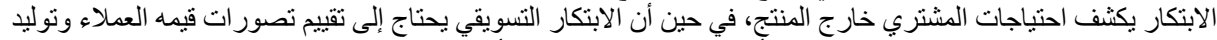

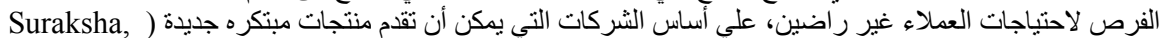

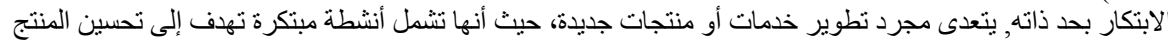

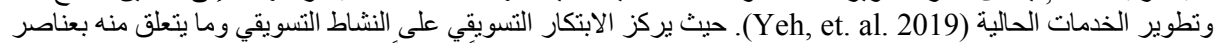

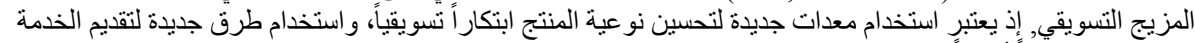

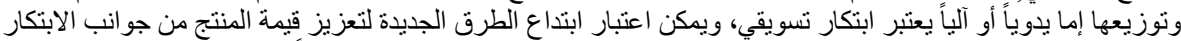

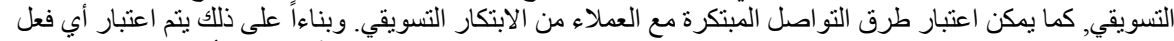

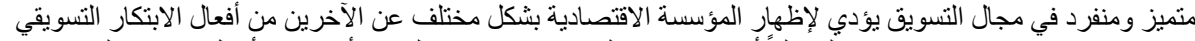

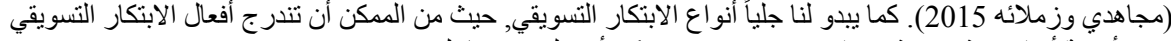

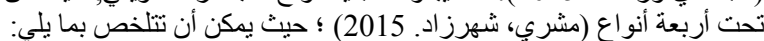
(1 الابتكار في المنتج.

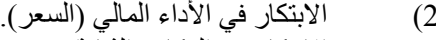

3 ) الابتكار في المكان (الثقافة).

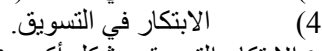

ولتوضيح أنواع الابتكار التسويقي بشّكل أكبر، نتطرق للابثديث عن كل نوع على حدا. (1 الابتكار في المنتج.

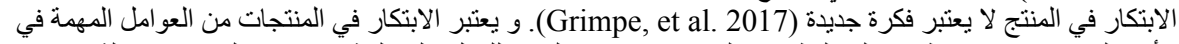

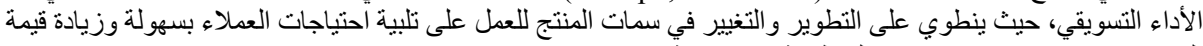

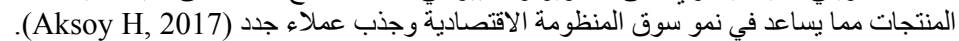
الابتكار في الأداء المالي (السعر).

(2

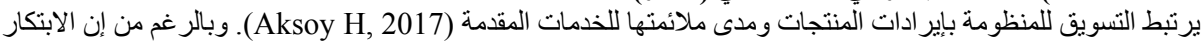

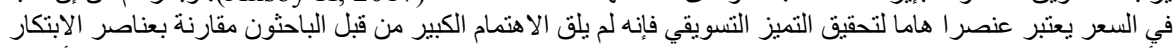

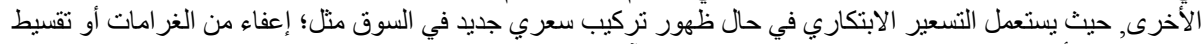

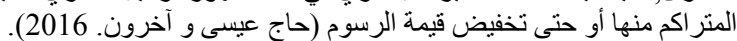
الابتكار في المكان (الثقافة).

(3

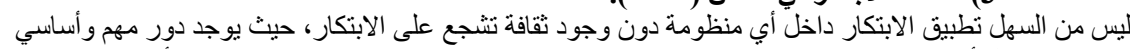

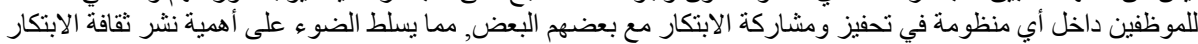

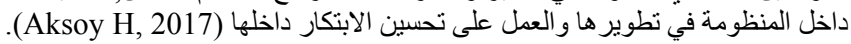

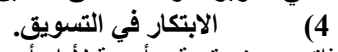

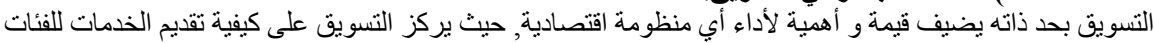

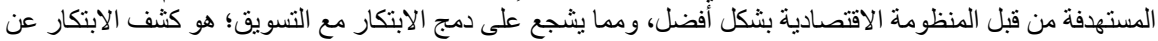

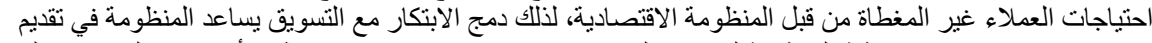

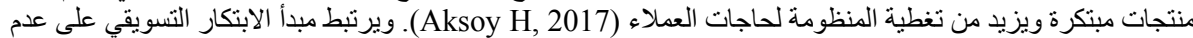

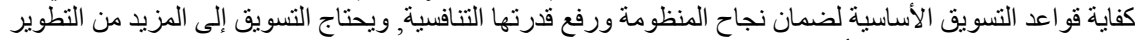

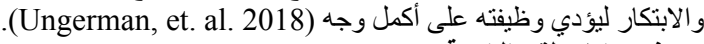
تعريف رضا متلقي الخدمة:

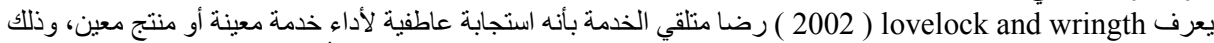

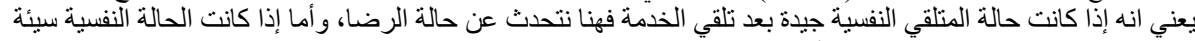

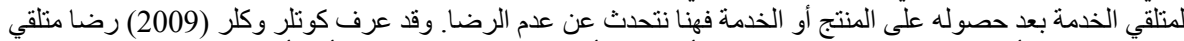

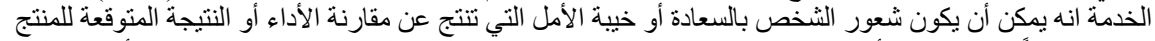

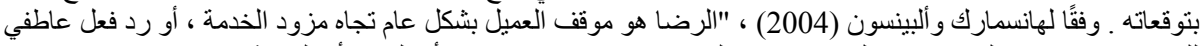

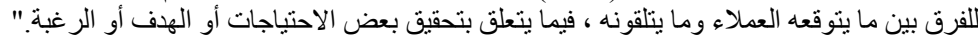

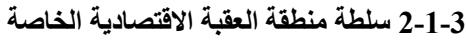

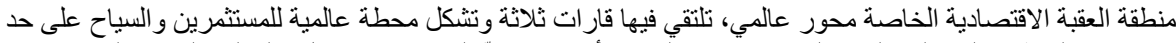

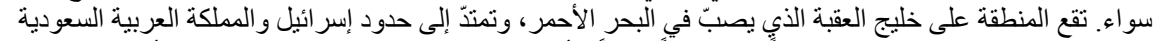

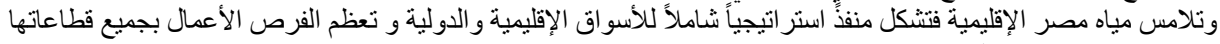
و توفر مستوى حياة منطور. 
تضطلع سلطة منطقة العقبة الإقتصادية الخاصة بدور رائد على الصعيد الإداري و الحكومي في المملكة الأردنية الهاشمية،

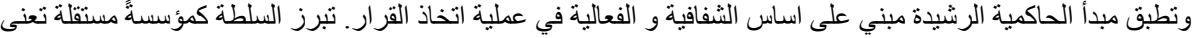

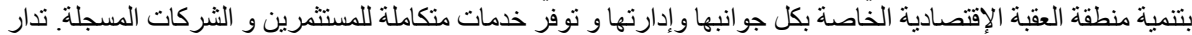

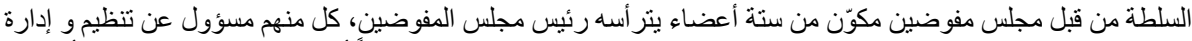

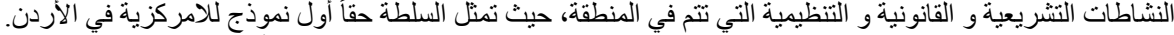

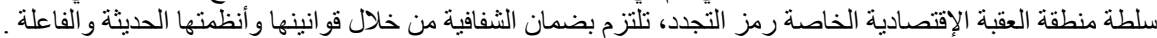
الأهداف المؤسسية للسلطة

ل $\quad$ لتمية المنطقة والإقليم و إيجاد بيئة استثمارية منطورة مؤهلة هلة لاستقطاب الاستثمار ات الصناعية والتجارية و السياحية و الخدماتيه.

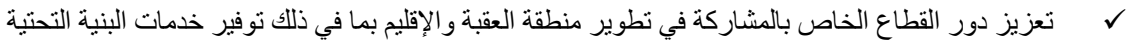

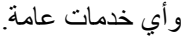
تشجيع المنافسة ومنع الاحتكار في الأنشطة الاقتصادية المختلفة داخل المنطقة.

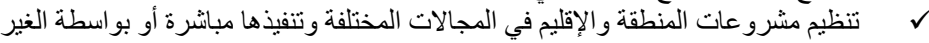

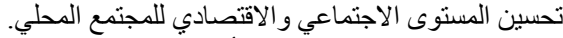

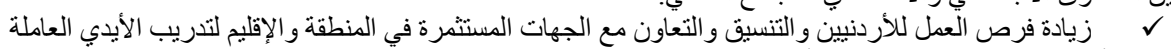

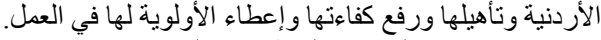
حماية البيئة في المنطقة و الإقليه.

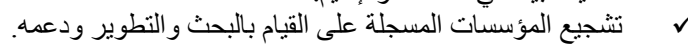

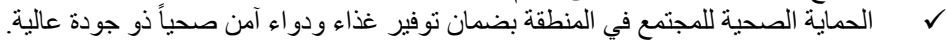

2-2 2-2 الار اسات السابقة:

2-2-1

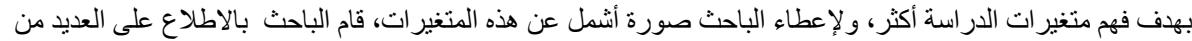

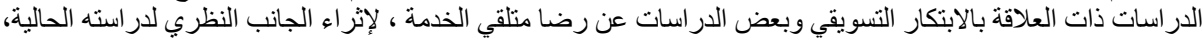

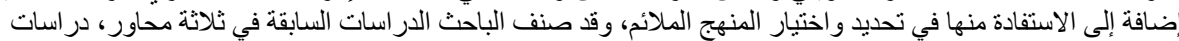

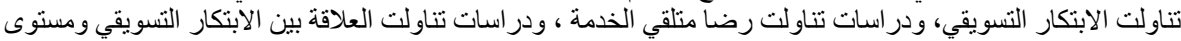

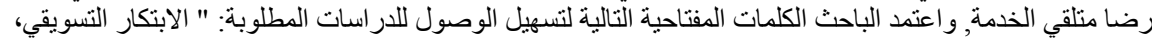
مستوى رضا متلقي الخدمة ". وفيما يلي استعر اض للإر اسات السابقة مصنفة تبعاً لمحاور البحث ومرتبةً من الأقدم إلى الأحدث: 2-2-2 الدراسات التي تناولت الابتكار التسويقي:

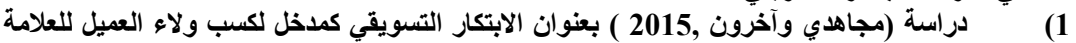

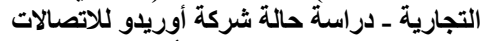

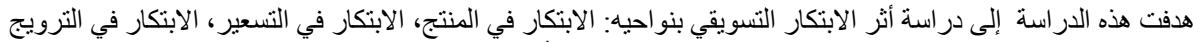

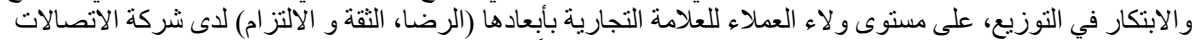

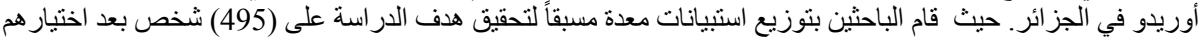

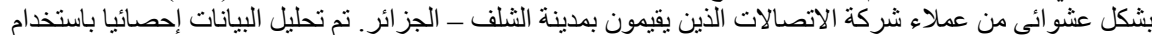

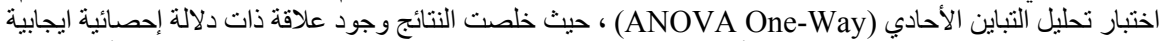

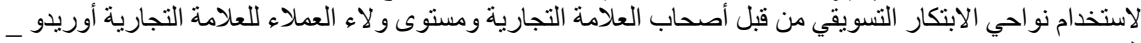

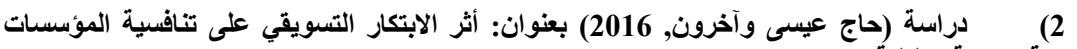
الجزائرية ـ دراسة ميدانية تحليلية التاجية

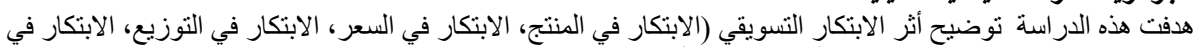

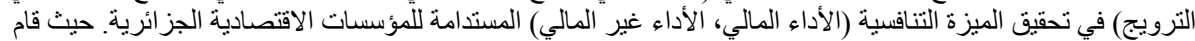

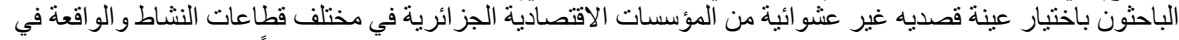

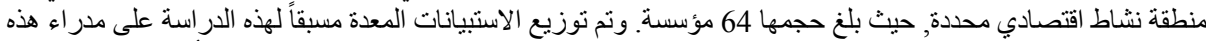

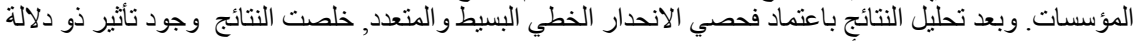

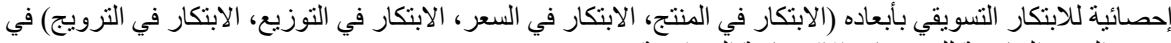
تحقيق الميزة التنافسية للمؤسسات الاقتصادية التادية الجز ائرية.

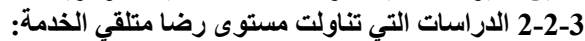

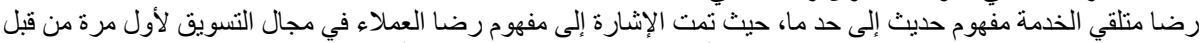

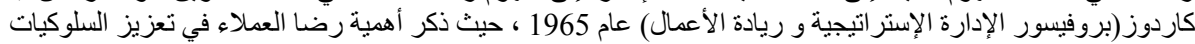

الاستهلاكية لديهم (Zheng, et al. 2017). 
Impact of Internet Banking Service Quality on Customer Satisfaction

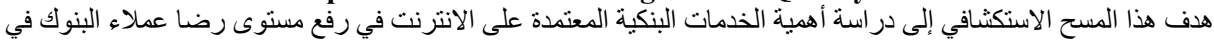

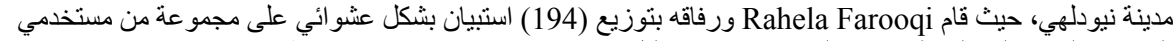

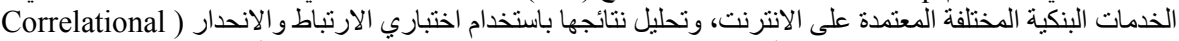

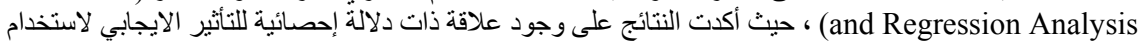

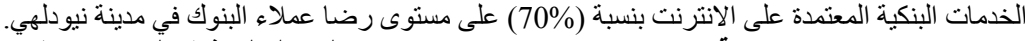

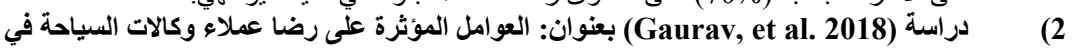

Factors Influencing Customer Satisfaction at Tours and Travel Agencies in India

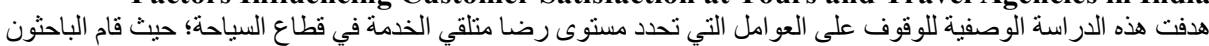

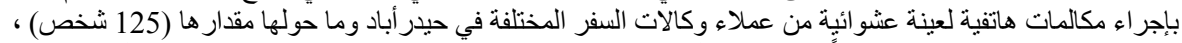

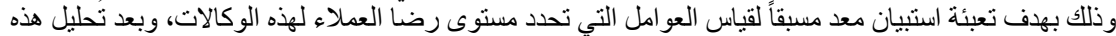

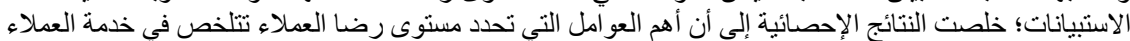

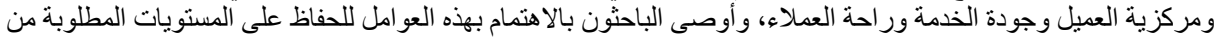

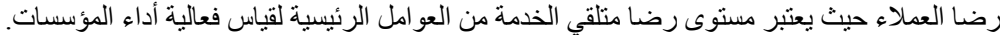

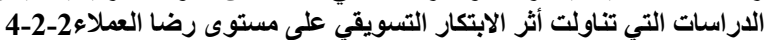

1

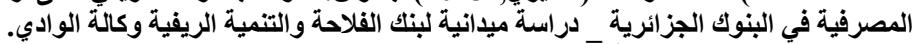

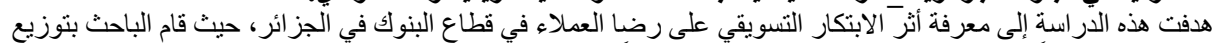

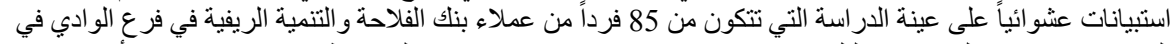

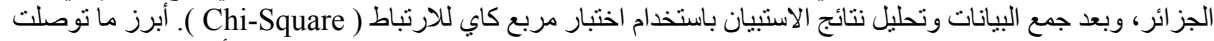

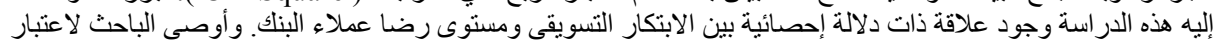

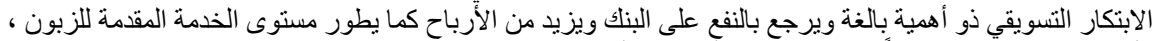

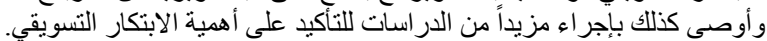

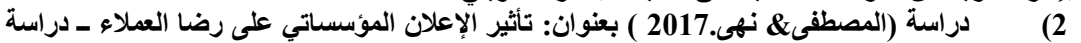

ميدانية على عملاء المصارف السورية

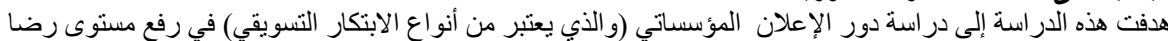

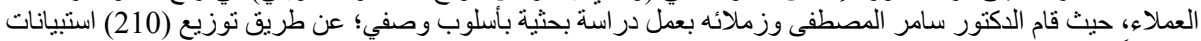

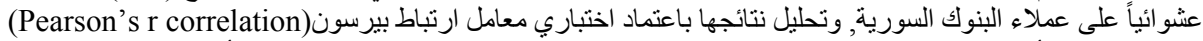

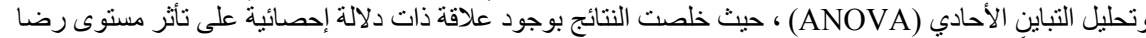

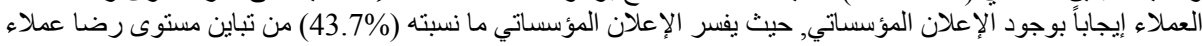
البنوك السورية. 3 منهجية الدراسة المنة

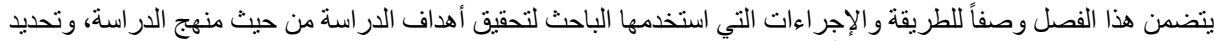

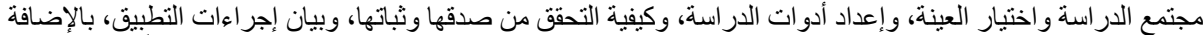

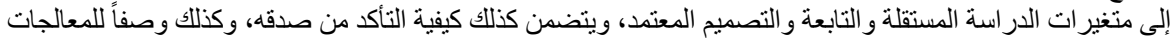

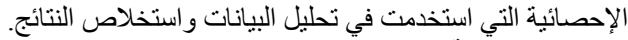

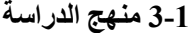
استندت الدارسة على المنهج الوصفي (لقياس مستوى رضا منلقي الخدمة) التحليلي ( لدراسة علاقة أثر الابتكار على مستوى

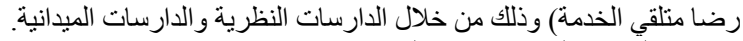

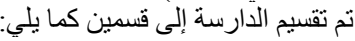

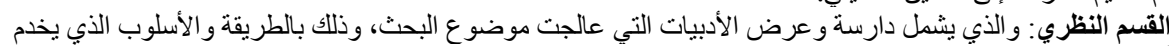

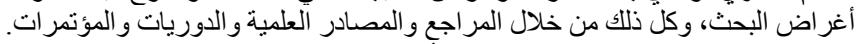

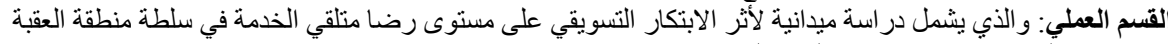
الاقتصادية الخاصة، نم إجر ائها في الفترة الو اقعة ما بين (2019/5/1) و (2019/5/15).

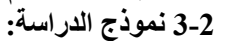

في ضوء فر فرذجيات الدراسة و المتغير ات المعتمدة فإن النموذج الفرضي للار اسة (من إعداد الباحث) هو كما يلي: 
The Impact of Marketing Innovation on Customer Satisfaction ....

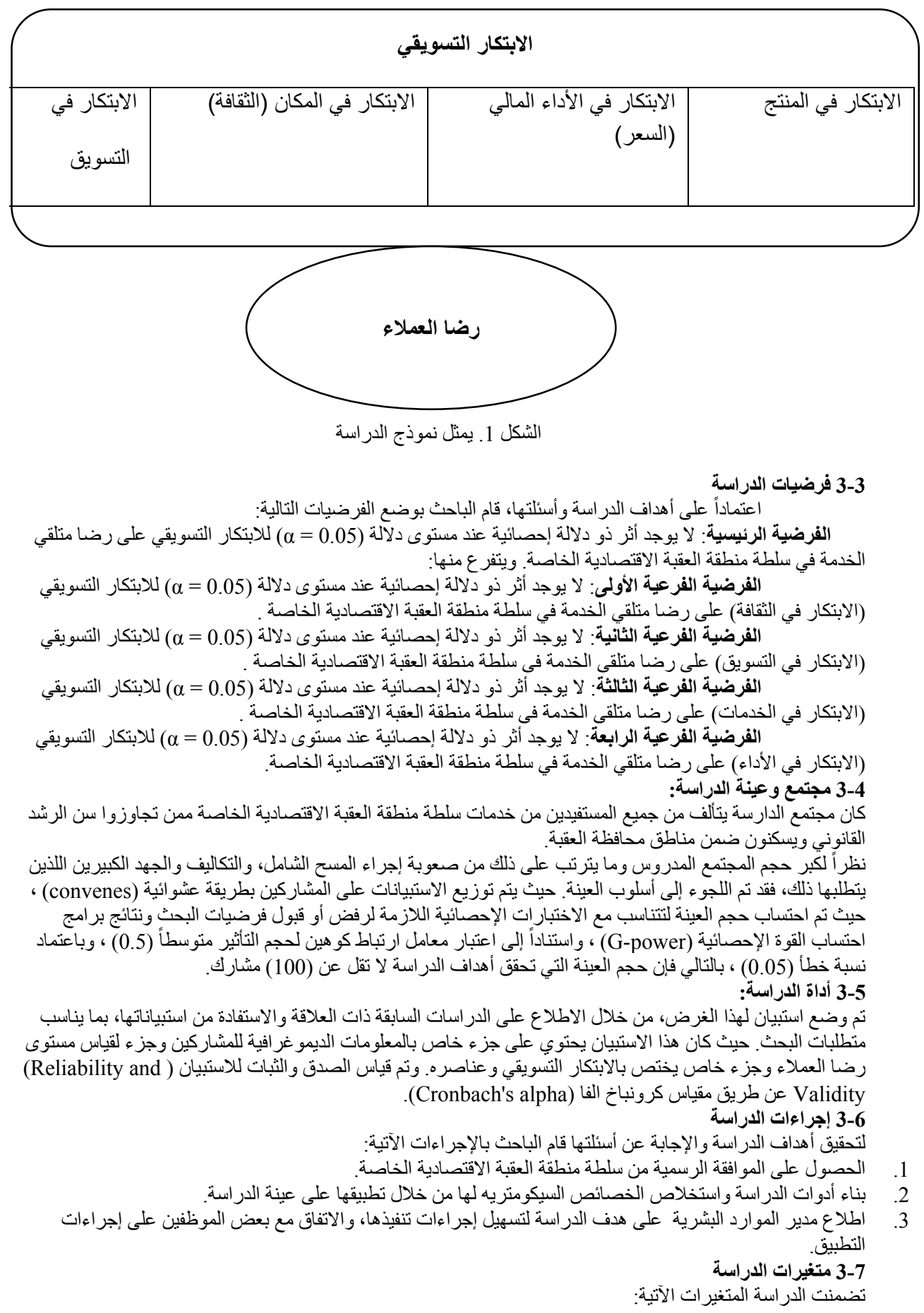




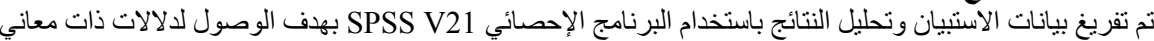

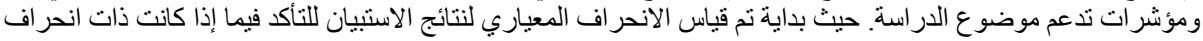

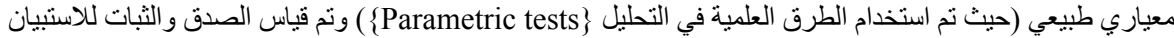
(Cronbach's alpha) عن طريق مقياس كرونباخ اللك قام الباحث بتحليل النتائج (Validity and Reliability)

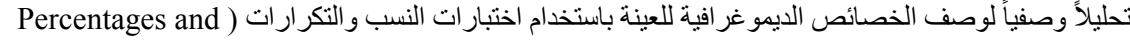
.(frequencies

وبهذف اختبار فرضيات الدر اسة، نم إجر اء اختبار معامل بيرسون للارتباط (Pearson-r ) لاختبار الفرضية الرئيسية والتي

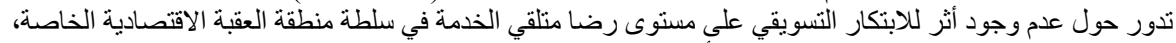

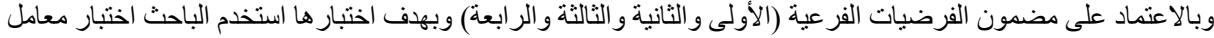

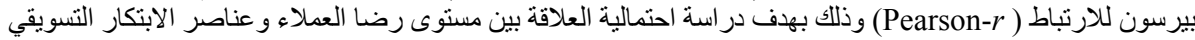
كل على حدا. نتائج الار اسة

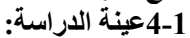

بهدف جمع البيانات من مجتمع الدر اسة، قام الباحث بتوزيع الاستبيانات على (110) من المستفيدين من خدمات سلطة منطقة

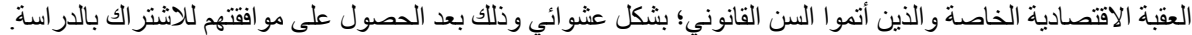

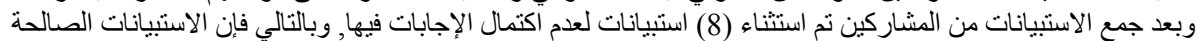

للتحليل بلغت (102) استبيان بنسبة استجابة بلغت (92.7\%). 4-2 نتائج الاستبيان: 2-2 وبع 4-1 وصف العينة

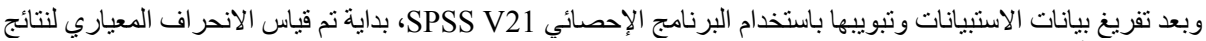

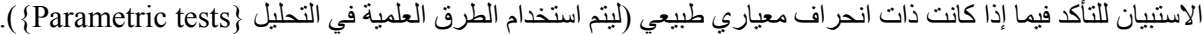

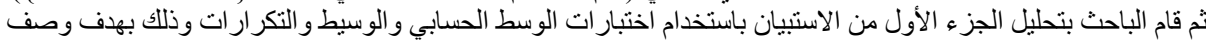

عينة الدراسة وتم تلخيص النتائج الديمو غر افية للعينة بالجدول رقم (1) :

الجدول رقم (1): المتغير ات الديمو غر افية لعينة الدراسة

\begin{tabular}{|c|c|c|c|}
\hline النسبة المئوية & التكر ار & المستوى & المتغير \\
\hline $63.7 \%$ & 65 & ذكر & \multirow[t]{2}{*}{ الجنس } \\
\hline $36.3 \%$ & 37 & أنثى & \\
\hline $45 \%$ & 46 & سنة25- 22 & \multirow{3}{*}{ العمر } \\
\hline $33 \%$ & 34 & سنة 26-30 & \\
\hline $22 \%$ & 22 & سنة 45-30 & \\
\hline $40.2 \%$ & 41 & ثانوي & \multirow[t]{4}{*}{ المستوى التعليمي } \\
\hline $47 \%$ & 48 & جامعي & \\
\hline $8 \%$ & 8 & در اسات عليا & \\
\hline $4.8 \%$ & 5 & لا شيء & \\
\hline $26.5 \%$ & 27 & موظف & \multirow[t]{4}{*}{ الوظيفة } \\
\hline $44 \%$ & 45 & مهن حرة & \\
\hline $23.5 \%$ & 24 & تاجر & \\
\hline $6 \%$ & 6 & أخرى & \\
\hline
\end{tabular}

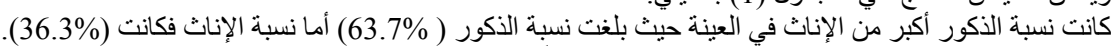

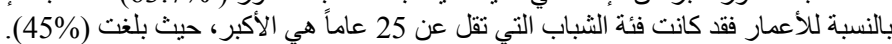

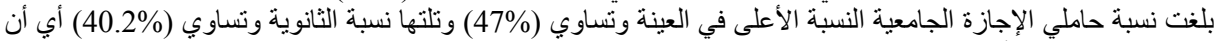
نسبة المتعلمين هي الأعلى في العينة.

أما بالنظر إلى الوظائف الخاصَّة بأفر اد العينة، حيث بلغت النسبة الأعلى لذوي المهن الحرة (44\%). 
4-2-2 قياس الصدق والثبات للاستبيان (Scale Validity and Reliability)

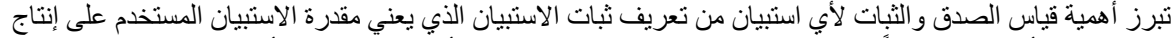

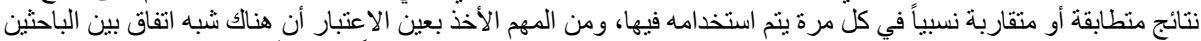

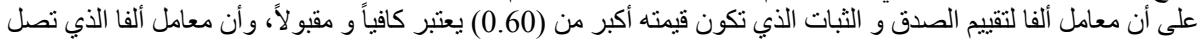

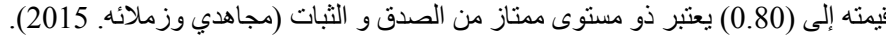

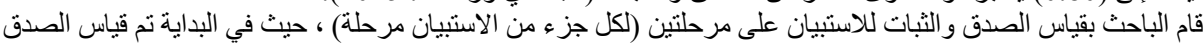

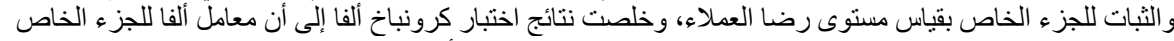

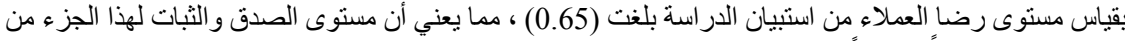

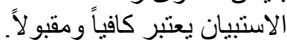

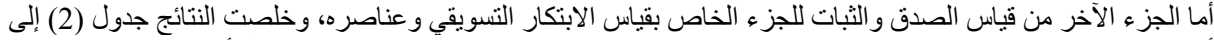

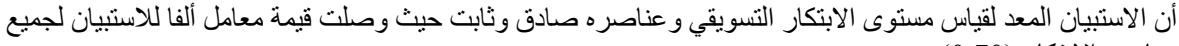

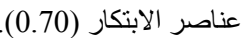

جدول رقم (2) : نتائج اختبار درجة الثبات ألفا لاستبيان الابتكار التسويقي

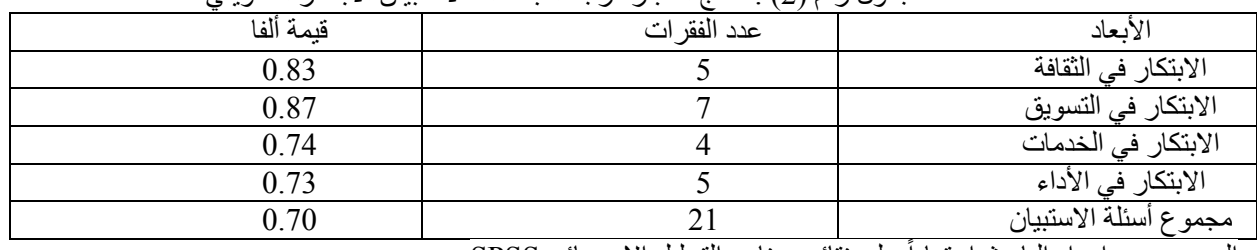

المصدر : من إعداد الباحث اعتماداً على نتائج برنامج التحليل الإحصائي

4-3 فرضيات الاراسة

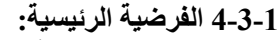

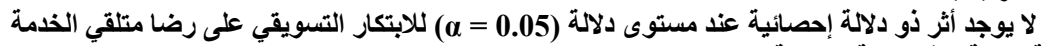

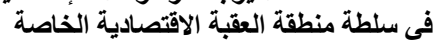

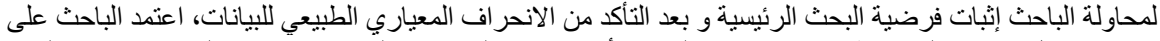

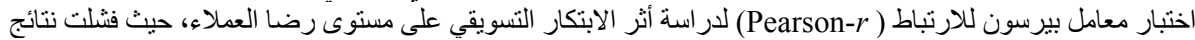

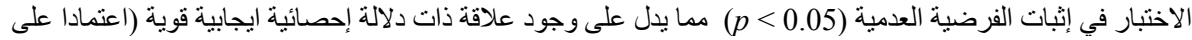

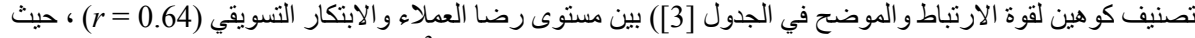

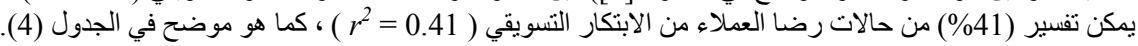

جدول رقم (3): دلالات نتائج اختبار الارتباط (Cohen, 1988)

\begin{tabular}{|c|c|}
\hline قيمة الدلالة (r) & قوة العلاقة \\
\hline $0.3-0.1$ & ضعيفة \\
\hline $0.5-0.3$ & متوسطة \\
\hline $1-0.5$ & قوية \\
\hline
\end{tabular}

جدول رقم (4): نتائج علاقة مستوى رضا العملاء مع الابتكار التسويقي

\begin{tabular}{|c|c|c|c|}
\hline & & مستوى رضا العملاء & الابتكار التسويقي \\
\hline \multirow[t]{3}{*}{ مستوى رضا العملاء } & Pearson Correlation & 1 & $0.64^{* *}$ \\
\hline & Sig. (2-tailed) & & 0.00 \\
\hline & $\mathrm{N}$ & & 102 \\
\hline \multirow[t]{3}{*}{ الابتكار التسويقي } & Pearson Correlation & $0.64^{* *}$ & 1 \\
\hline & Sig. (2-tailed) & 0.00 & \\
\hline & $\mathrm{N}$ & 102 & \\
\hline
\end{tabular}

المصدر : من إعداد الباحث اعتماداً على نتائج برنامج التحليل الإحصائي SPSS 
4-3-2 - 2 الفرضية الفرعية الأولى:

لا يوجد أثر ذو دلالة إحصائية عند مستوى دلالة (م)

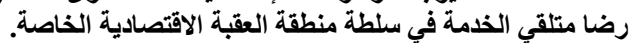

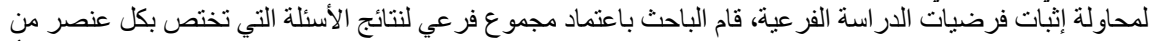

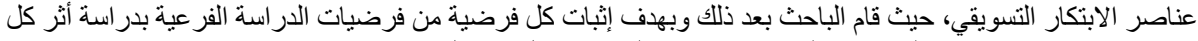

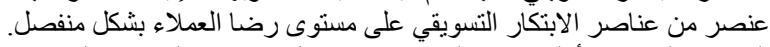

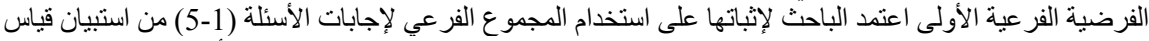

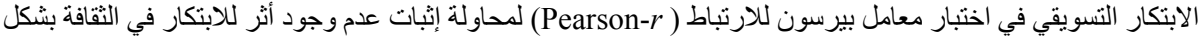

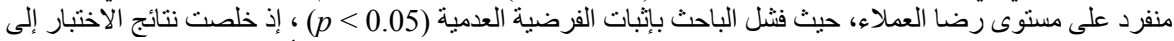

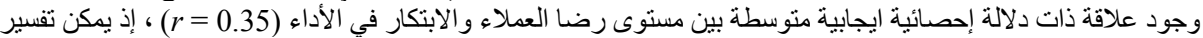

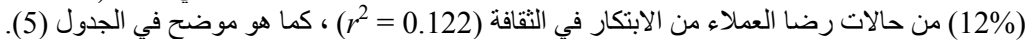
جدول رقم (5): نتائج علاقة مستوى رضاف رضا العملاء مع الابتكار في الثنافة

\begin{tabular}{|c|c|c|c|}
\hline & & مستوى رضا العملاء & الابتكار في الثقافة \\
\hline \multirow[t]{3}{*}{ مستوى رضا العملاء } & Pearson Correlation & 1 & $0.35^{* \prime \prime}$ \\
\hline & Sig. (2-tailed) & & 0.00 \\
\hline & $\mathrm{N}$ & & 102 \\
\hline \multirow[t]{3}{*}{ الابتكار في الثقافة } & Pearson Correlation & $0.35^{* *}$ & 1 \\
\hline & Sig. (2-tailed) & 0.00 & \\
\hline & $\mathrm{N}$ & 102 & \\
\hline
\end{tabular}
SPSS المصدر: من إعداد الباحث اعتماداً على نتائج برنامج التحليل الإحصائي الفئي

4-3-3 الفرضية الفرعية الثانية:

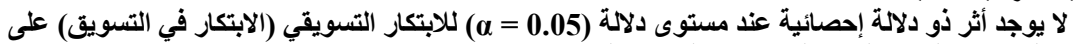

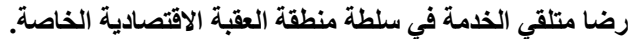

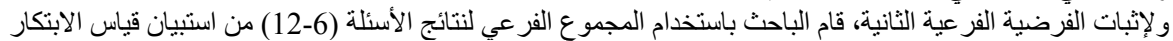

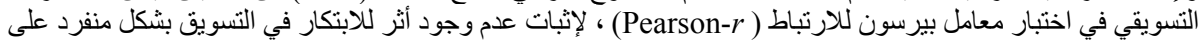

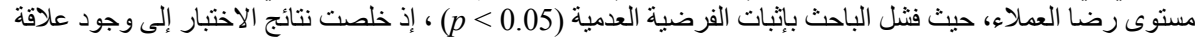

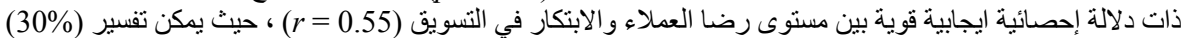

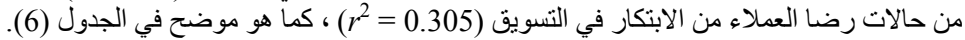

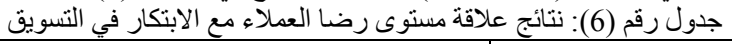

\begin{tabular}{|c|c|c|c|}
\hline & & مستوى رضا العملاء & الابتكار في التسويق \\
\hline \multirow[t]{3}{*}{ مستوى رضا العملاء } & Pearson Correlation & 1 & $0.55^{* *}$ \\
\hline & Sig. (2-tailed) & & 0.00 \\
\hline & $\mathrm{N}$ & & 102 \\
\hline \multirow[t]{3}{*}{ الابتكار في التسويق } & Pearson Correlation & $0.55^{* *}$ & 1 \\
\hline & Sig. (2-tailed) & 0.00 & \\
\hline & $\mathrm{N}$ & 102 & \\
\hline
\end{tabular}

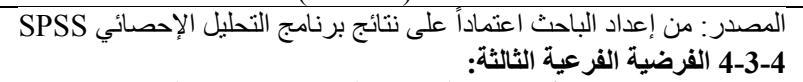

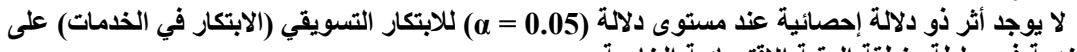

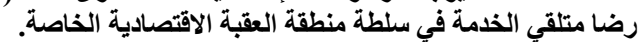

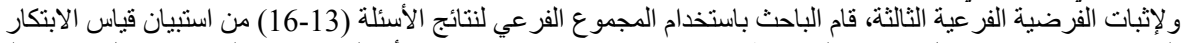

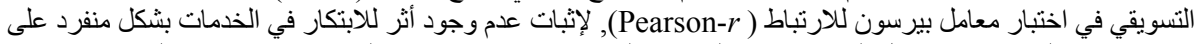

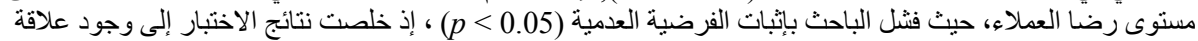

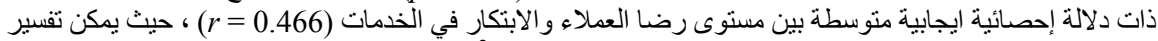

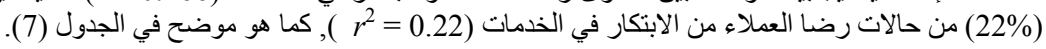


The Impact of Marketing Innovation on Customer Satisfaction ....

\begin{tabular}{|c|c|c|c|}
\hline & & مستوى رضا العملاء & الابتكار في الخدمات \\
\hline \multirow[t]{3}{*}{ مستوى رضا العملاء } & Pearson Correlation & 1 & $0.466^{* *}$ \\
\hline & Sig. (2-tailed) & & 0.00 \\
\hline & $\mathrm{N}$ & & 102 \\
\hline \multirow[t]{3}{*}{ الابتكار في الخدمات } & Pearson Correlation & $0.466^{* *}$ & 1 \\
\hline & Sig. (2-tailed) & 0.00 & \\
\hline & $\mathrm{N}$ & 102 & \\
\hline \multicolumn{4}{|c|}{ **. Correlation is significant at the 0.01 level (2-tailed). } \\
\hline \multicolumn{4}{|c|}{ 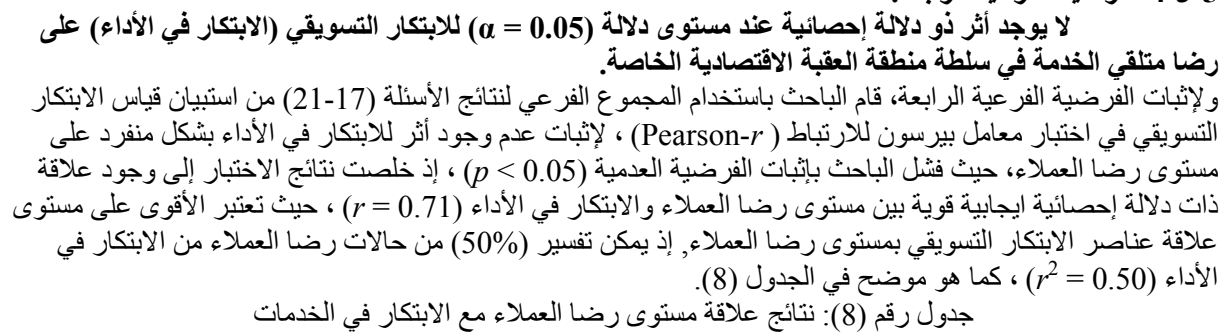 } \\
\hline & & مستوى رضا العملاء & الابتكار في الاداء \\
\hline \multirow[t]{3}{*}{ مستوى رضا العملاء } & Pearson Correlation & 1 & $0.71^{* *}$ \\
\hline & Sig. (2-tailed) & & 0.00 \\
\hline & $\mathrm{N}$ & & 102 \\
\hline \multirow[t]{3}{*}{ الابتكار في الاداء } & Pearson Correlation & $0.71^{* *}$ & 1 \\
\hline & Sig. (2-tailed) & 0.00 & \\
\hline & $\mathrm{N}$ & 102 & \\
\hline \multicolumn{4}{|c|}{ **. Correlation is significant at the 0.01 level (2-tailed). } \\
\hline \multicolumn{4}{|c|}{ 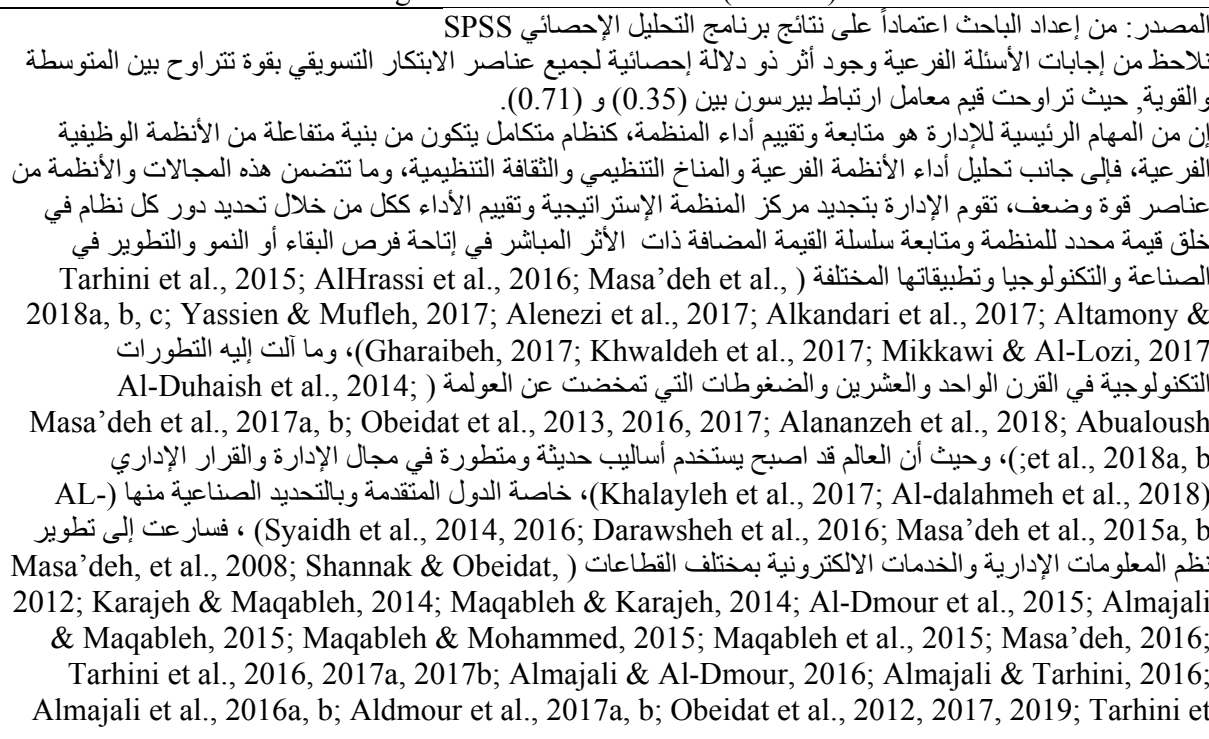 } \\
\hline
\end{tabular}




\section{Journal of Social Sciences (COES\&RJ-JSS), 8(3), pp.399-417}

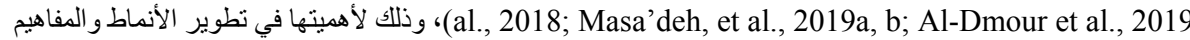

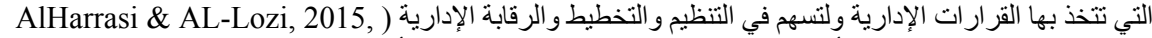

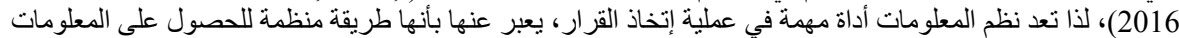

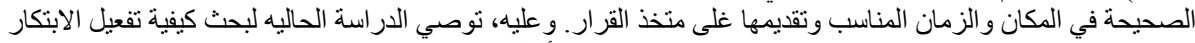
التسويقي واستثمار نظم المعلومات الإدارية المنطورة والحيثة التيثة من أجل تحقيق التميز المنظمي المنشود.

مناقشة النتائج والتوصيات

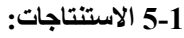

يوجد علاقة ذات دلالة إحصائية بين مستوى رضا العملاء و الابتكار التسويقي لدى منلقي الخدمة لدى سلطة العقبة الاقتصادية الخاصة.

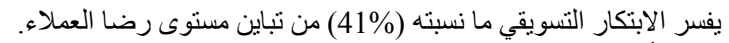

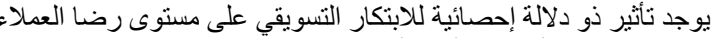

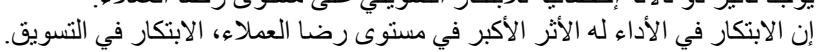

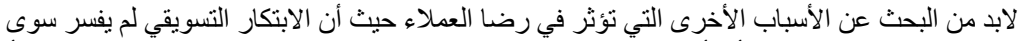
5-2

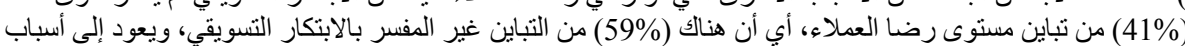

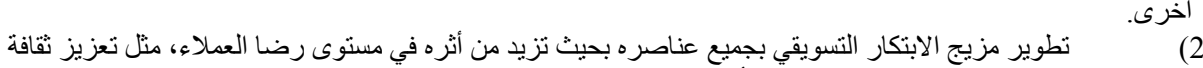
الابتكار وتطوير الابتكار في الخدمات المقدمة أكثر.

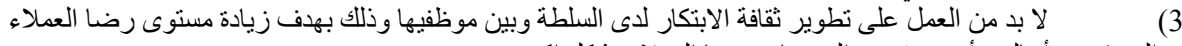

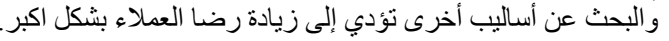

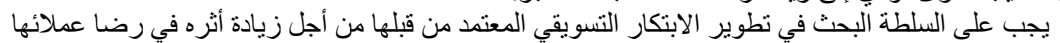
و الاستفادة في ذلك من تجربة المؤسسات الحكومية الأخرى.

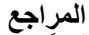

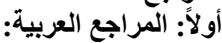

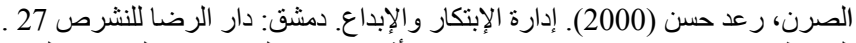

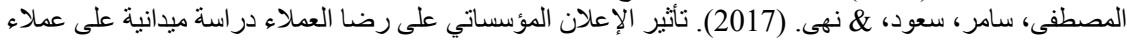

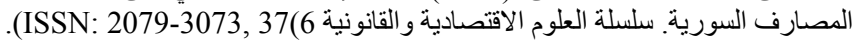

حاج عيسى، سيد، مجاهدي \& فاتح، اير اين \& إيمان. (2016). اثر الابتكار التسويقي على تنافسية المؤسسات الجزائرية در اسة ميدانية تحليلية.

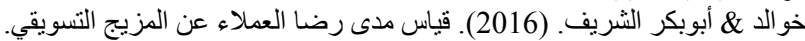

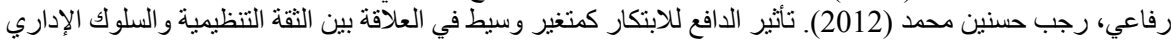
الابتكاري "دراسة ميدانية بالتطبيق على المديرين العاملين بالجهاز الإداري بجامعة الطائف". المجلة العلمية، 9 (52)، 78-

رماح (2016). أثر الابتكار التسويقي على تنافسية المؤسسات الجزائرية ـ دراسة ميدانية تحليلية، المؤتمر العلمي الدولي

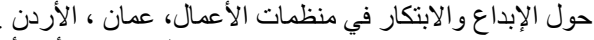

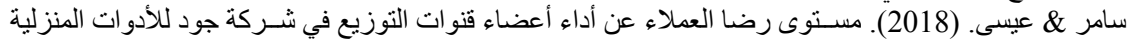

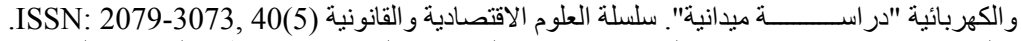

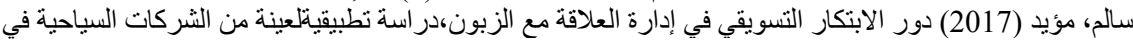

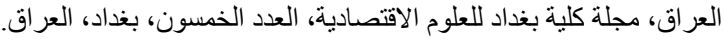

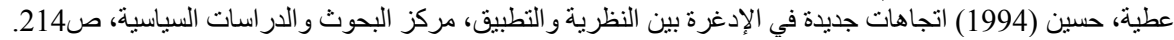

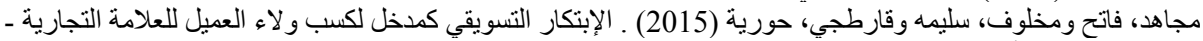

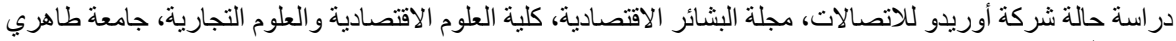

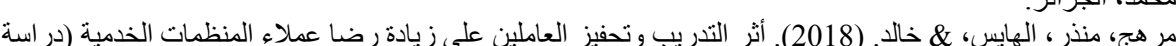

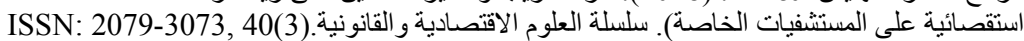
مشري (2015) ـأثر الابتكار التسويقي على رضات العهاء العملاء عن جودة الخدمة المصرفية في البنوك الجز ائرية، رسالة ماجستير

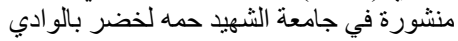
مصطفاوي \& ع عبد الرؤوف. (2017). دور نظام المعلومات التسويقية في تحقيق رضا العملاء در اسة حالة مؤسسة مو بيليس

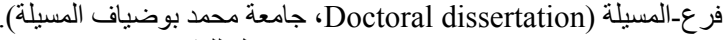
نجم، نجم (2003). ادارة الإبتكار . عمان: دار و ائل للنشر. 
نصور، ريزان، \& حماد. (2018). تأثير الإعلان عبر مو اقع التو اصل الاجتماعي في رضا المستهلك على لعلامة التجارية

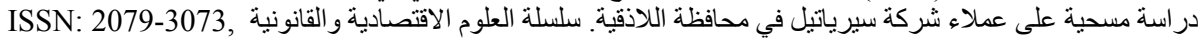
. $39(4$

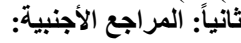

\section{References}

Abualoush, S., Bataineh, K., \& Alrowwad, A. (2018a). The Role of Knowledge Management Process and Intellectual Capital as Intermediary Variables between Knowledge Management Infrastructure and Organization Performance. Interdisciplinary Journal of Information, Knowledge, and Management, 13, 279-309.

Abualoush, S., Obeidat, B., \& Tarhini, A. (2018b). The Role of Employees' Empowerment as an Intermediary Variable between Knowledge Management and Information Systems on Employees' Performance. VINE Journal of Information and Knowledge Management Systems, 48(2), 217-237.

Alenezi, H., Tarhini, A., Alalwan, A., \& Al-Qirim, N. (2017). Factors Affecting the Adoption of EGovernment in Kuwait: A Qualitative Study. Electronic Journal of e-Government, 15(2), 84-102.

Alananzeh, O., Jawabreh, O., Al-Mahmoud, A., \& Hamada, R. (2018). The Impact of Customer Relationship Management on Tourist Satisfaction: The Case of Radisson Blue Resort in Aqaba City. Journal of Environmental Management and Tourism, 2(26), 227-240.

Al-dalahmeh, M., Khalaf, R., \& Obeidat, B. (2018). The Effect of Employee Engagement on Organizational Performance via the Mediating Role of Job Satisfaction: The Case of IT Employees in Jordanian Banking Sector. Modern Applied Science, 12(6), 17-43.

Al-Dmour, R., Al Haj Dawood, E., Al-Dmour, H., \& Masa'deh, R. (2019). The Effect of Customer Lifestyle Patterns on the Use of Mobile Banking Applications in Jordan. Int. J. Electronic Marketing and Retailing, Forthcoming.

Aldmour, R., Hammdan, F., Dmour, H., Alrowwad, A., \& Khwaldeh, S. (2017a). The Effect of Lifestyle on Online Purchasing Decision for Electronic Services: The Jordanian Flying E-Tickets Case. Asian Social Science, 13(11), 157-169.

Aldmour, R., Masa'deh, R., \& Obeidat, B. (2017b). Factors Influencing the Adoption and Implementation of HRIS Applications: Are they Similar. International Journal of Business Innovation and Research, 14(2), 139-167.

Al-Dmour, R, Obeidat, B., \& Almajali, D. (2015). The Practice of HRIS Applications in Business Organizations in Jordan: An Empirical Study. 4th Scientific \& Research Conference on New Trends in Business, Management and Social Sciences (COES\&RJ-TK15/1).

Al-Duhaish, A., Alshurideh, M., \& Al-Zu'bi, Z. (2014). The Impact of the Basic Reference Group Usage on the Purchasing Decision of Clothes (A Field Study of Saudi Youth in Riyadh City). Dirasat: Administrative, 41(2), 205-221.

Alenezi, H., Tarhini, A., Alalwan, A., \& Al-Qirim, N. (2017). Factors Affecting the Adoption of EGovernment in Kuwait: A Qualitative Study. Electronic Journal of e-Government, 15(2), 84-102. 
AlHarrasi, J., \& AL-Lozi, M. (2015). The Role of Innovation Management and Technological Innovation on Organizational Effectiveness. 4th Scientific \& Research Conference on New Trends in Business, Management and Social Sciences (COES\&RJ-TK15/1), Istanbul, Turkey.

AlHarrasi, J., \& AL-Lozi, M. (2016). The Role of Innovation Management and Technological Innovation on Organizational Effectiveness: A Theoretical Model. Journal of Social Sciences (COES\&RJ-JSS), 5(1), 80-95.

AlHrassi, J., Al-Lozi, M., \& Irtaimeh, H. (2016). The Impact of Management Innovation and Technological Innovation on Organizational Effectiveness: An Empirical Study from Managerial Staff Perspective in Sultan Qaboos University. Journal of Social Sciences (COES\&RJ-JSS), 5(3), 309-339.

Alkandari, A., Masa'deh, R., \& Al-Lozi, M. (2017). Knowledge Management and its Role on Organizational Crisis Management: A Literature Review. Journal of Social Sciences (COES\&RJJSS), 6(4), 833-850.

Aksoy, H. (2017). How do Innovation Culture, Marketing Innovation and Product Innovation Affect the Market Performance of Small and Medium-Sized Enterprises (SMEs)?, Technology in Society doi: 10.1016/j.techsoc.2017.08.005.

Ali, Ari, M. (2017). The Effect of Marketing Innovation on The Dimensions of Consumer Behavior Based on Internal Factors: A Survey Study of The Opinions of a Sample of Iphone Users in Duhok City. Academic Journal of Nawroz University, 6(2), 251-273.

Almajali, D., \& Al-Dmour, R. (2016). The Role of Information Technology in Motivating Students to Accept E-Learning Adoption in Universities: A Case Study in Jordanian Universities. Journal of Business \& Management (COES\&RJ-JBM), 4(1), 36-46.

Almajali, D., \& Maqableh, M. (2015). Assessing the Digital Divide Status of the Jordanian Telecentre. International Journal of Communications, Network and System Sciences, 8(11), 428-439.

Almajali, D., \& Tarhini, A. (2016). Antecedents of ERP Systems Implementation Success: A Study on Jordanian Healthcare Sector. Journal of Enterprise Information Management, 29(4), 549565.

Almajali, D., Mansour, K., \& Maqableh, M. (2016a). The Impact of Electronic Supply Chain Management Usage on Firm's Performance. International Journal of Communications, Network and System Sciences, 9(6), 280-293.

Almajali, D., Masa'deh, R., \& Al-Lozi, M. (2016b). Determinants of the Actual Use of E-Learning Systems: An Empirical Study on Zarqa University in Jordan. Journal of Social Sciences (COES\&RJ-JSS), 5(2), 172-200.

AL-Syaidh, N., Al- Lozi, M., \& AlHarrasi, J. (2016). Transformational Leadership and its Role on the Effectiveness of Employees' Behavior: A Theoretical Study. Journal of Business \& Management (COES\&RJ-JBM), 4(1), 14-35. 
AL-Syaidh, N., Masa'deh, R., \& Al-Zu'bi, Z. (2014). Transformational Leadership and its Impact on the Effectiveness of Employees' Behavior in the Public and Private Jordanian Hospitals. Jordan Journal of Business Administration, 11(1), 23-57.

Altamony, H., \& Gharaibeh, A. (2017). The Role of Academic Researcher to Mintzberg's Managerial Roles. International Journal of Business Management and Economic Research, 8(2), 920-925.

Altobishi, T., Erboz, G., \& Podruzsik, S. (2018). E-Banking Effects on Customer Satisfaction: The Survey on Clients in Jordan Banking Sector. International Journal of Marketing Studies, 10(2), 151-161.

Bismo, A., Sarjono, H., \& Ferian, A. (2018). The Effect of Service Quality and Customer Satisfaction on Customer Loyalty: A Study of Grabcar Services in Jakarta. Pertanika Journal of Social Sciences \& Humanities.

Blayse, M., \& Manley, K. (2004). Key Influences on Construction Innovation, Construction Innovation, 4, 143-154.

Bodemann, M. (2015). Risk Awareness after The Adoption of New Steering Model in German Public Administrations -A Case Study, 2nd Global Conference on Business, Economics, Management and Tourism, Volume, 23.

Darawsheh, S., ALshaar, A., \& AL-Lozi, M. (2016). The Degree of Heads of Departments at the University of Dammam to Practice Transformational Leadership style from the Point of View of the Faculty Members. Journal of Social Sciences (COES\&RJ-JSS), 5(1), 56-79.

Firdous, S., \& Farooqi, R. (2017). Impact of Internet Banking Service Quality on Customer Satisfaction. The Journal of Internet Banking and Commerce, 22(1), 1-17.

Gaurav, K., Sahu, K.C., \& Mathew, S. (2018). Factors Influencing Customer Satisfaction at Tours and Travel Agencies in India. International Journal of Research in Management, Economic and Commerce, 8(3), 31-38.

Grimpe, C., Sofka, W., Bhargava, M., \& Chatterjee, R. (2017). R\&D, Marketing Innovation, and New Product Performance: A Mixed Methods Study. Journal of Product Innovation Management, 34(3), 360-383.

Karajeh, H., \& Maqableh, M. (2014). Security of Cloud Computing Environment. The 23rd IBIMA Conference on Vision 2020: Sustainable Growth, Economic Development, and Global Competitiveness, USA, 2202-2215.

Khalayleh, W., Masa'deh, R., \& Al-Lozi, M. (2017). Administrative Empowerment and its Role on the Work Teams Performance: A Literature Review. Journal of Social Sciences (COES\&RJJSS), 6(4), 851-868. 
Khwaldeh, S., Al-Hadid, I., Masa'deh, R., \& Alrowwad, A. (2017). The Association between EServices Web Portals Information Quality and ICT Competence in the Jordanian Universities. Asian Social Science, 13(3), 156-169.

Maier, A., \& Dan, H. (2018). Influence of the Marketing Innovation on the Organizational Performance. International Journal of Advanced Engineering and Management Research, 3(6), 182-192.

Maqableh, M., \& Mohammed, A. (2015). The Acceptance and Use of Computer based Assessment in Higher Education. Journal of Software Engineering and Applications, 8(10), 557.

Maqableh, M., \& Karajeh, H. (2014). Job Scheduling for Cloud Computing using Neural Networks. Communications and Network, 6(3), 191-200.

Maqableh, M., Rajab, L., Quteshat, W., Khatib, T., \& Karajeh, H. (2015). The Impact of Social Media Networks Websites Usage on Students' Academic Performance. Communications and Network, 7(4), 159-171.

Masa'deh, R. (2016). The Role of Knowledge Management Infrastructure in Enhancing Job Satisfaction at Aqaba Five Star Hotels in Jordan. Communications and Network, 8(4), 219-240.

Masa'deh, R., Alananzeh, O., Algiatheen, N., Ryati, R., Albayyari, R., \& Tarhini, A. (2017a). Employee's Perception of Implementing Green Supply Chain Management on Hotel's Economic and Operational Performance. Journal of Hospitality and Tourism Technology, 8(3), 395-416.

Masa'deh, R., Shannak, R., Maqableh, M., \& Tarhini, A. (2017b). The Impact of Knowledge Management on Job Performance in Higher Education: The Case of the University of Jordan. Journal of Enterprise Information Management, 30(2), 244-262.

Masa'deh, R., Alananzeh, O., Algudah, O., \& Tarhini, A. (2018a). The Effect of Promotional Mix on Hotel Performance during the Political Crisis in the Middle East. Journal of Hospitality and Tourism Technology, 9(1), 33-49.

Masa'deh, R., Alrowwad, A., Alkhalafat, F., Obeidat, B., \& Abualoush, S. (2018b). The Role of Corporate Social Responsibility in Enhancing Firm Performance from the Perspective of IT Employees in Jordanian Banking Sector: The Mediating Effect of Transformational Leadership. Modern Applied Science, 12(7), 1-26.

Masa'deh, R., Al-Henzab, J., Tarhini, A., \& Obeidat, B. (2018c). The Associations among Market Orientation, Technology Orientation, Entrepreneurial Orientation and Organizational Performance. Benchmarking: An International Journal, 25(8), 3117-3142.

Masa'deh, R., Hunaiti, Z., \& Bani Yaseen, A. (2008). An Integrative Model Linking IT-Business Strategic Alignment and Firm Performance: The Mediating Role of Pursuing Innovation and Knowledge Management Strategies. Communications of the International Business Information Management Association (IBIMA) Journal. 
Masa'deh, R., Gharaibeh, A., Tarhini, A., \& Obeidat, O. (2015a). Knowledge Sharing Capability: A Literature Review. 4th Scientific \& Research Conference on New Trends in Business, Management and Social Sciences (COES\&RJ-TK15/1), Istanbul, Turkey.

Masa'deh, R., Obeidat, B., Zyod, D., \& Gharaibeh, A. (2015b). The Associations among Transformational Leadership, Transactional Leadership, Knowledge Sharing, Job Performance, and Firm Performance: A Theoretical Model. Journal of Social Sciences (COES\&RJ-JSS), 4(2), 848-866.

Masa'deh, R., Almajali, D., Alrowwad, A., \& Obeidat, B. (2019a). The Role of Knowledge Management Infrastructure in Enhancing Job Satisfaction: A Developing Country Perspective. Interdisciplinary Journal of Information, Knowledge, and Management, 14, 1-25.

Masa'deh, R., Obeidat, B., Maqableh, M., \& Shah, M. (2019b). The Impact of Business Intelligence Systems on an Organization's Effectiveness: The Role of Metadata Quality from a Developing Country's View. International Journal of Hospitality \& Tourism Administration, Forthcoming.

Mckinley, W., Latham, S., \& Braun, M. (2014). Organizational Decline and Innovation: Turnarounds and Downward Spirals. Academy of Management Review, 39(1), 88-110.

Mikkawi, B., \& Al-Lozi, M. (2017). The Impact of Knowledge Management Infrastructure on Academic Staff Effectiveness: An Empirical Study at The University of Jordan. Jordan Journal of Business Administration, 13(1), 95-127.

Na, Y.K., Kang, S., \& Jeong, H.Y. (2019). The Effect of Market Orientation on Performance of Sharing Economy Business: Focusing on Marketing Innovation and Sustainable Competitive Advantage. Sustainability, 11(3), 729.

Obeidat, O., Al-Suradi, M., \& Tarhini, A. (2016). The Impact of Knowledge Management on Innovation: An Empirical Study on Jordanian Consultancy Firms. Management Research Review, 39(10), 1214-1238.

Obeidat, O., El-Rimawi, S., Maqableh, M., \& Al-Jarrah, I. (2013). Evaluating the Profitability of the Islamic Banks in Jordan. European Journal of Economics, Finance and Administrative Sciences, 56, 27-36.

Obeidat, O., Hadidi, A., \& Tarhini, A. (2017). Factors Affecting Strategy Implementation: A Case Study of Pharmaceutical Companies in the Middle East. Review of International Business and Strategy, 27(3), 386-408.

Obeidat, O., Tarhini, A., \& Aqqad, N. (2019). The Relationship among Emotional Intelligence, Conflict Management Styles, and Job Performance in Jordanian Banks. International Journal of Human Resources Development and Management, Forthcoming.

Obeidat, O., Sweis, R., Zyod, D., \& Alshurideh, M. (2012). The Effect of Perceived Service Quality on Customer Loyalty in Internet Service Providers in Jordan. Journal of Management Research, 4(4), 224-242. 
Omar, M. (2018). Elements of Marketing Innovation and its Impact on Marketing Performance/ Exploratory Study on a Sample of Individuals Working a Number of Commercial Companies in the City of Zakho. Humanities Journal of University of Zakho, 6(3), 870-888.

Oxford Dictionary of Business and Management, (2009), Oxford University Press, 5 Edition.

Shannak, R., \& Obeidat, B. (2012). Culture and the Implementation Process of Strategic Decisions in Jordan. Journal of Management Research, 4 (4), pp. 257-281.

Sulistyo, Heru (2016). Innovation Capability of SMEs through Entrepreneurship, Marketing Capability, Relational Capital and Empowerment, Marketing Capability, Relational Capital and Empowerment. Asia Pacific Management.

Suraksha Gupta, Naresh K. Malhotra, Michael Czinkota, \& Pantea Foroudi (2016). Marketing Innovation: A Consequence of Competitiveness. Journal of Business Research, 69(2016), 56715681.

Tarhini, A., Alalwan, A., Al-Qirim, N., \& Algharabat, R. (2018). An Analysis of the Factors Influencing the Adoption of Online Shopping. International Journal of Technology Diffusion (IJTD), 9(3), 68-78.

Tarhini, A., Al-Badi, A., Almajali, M., \& Alrabayaah, S. (2017a). Factors Influencing Employees' Intention to Use Cloud Computing. Journal of Management and Strategy, 8(2), 47.

Tarhini, A., Al-Busaidi, K., Bany Mohammed, A., \& Maqableh, M. (2017b). Factors Influencing Students' Adoption of E-Learning: A Structural Equation Modeling Approach. Journal of International Education in Business, 10(2), 164-182.

Tarhini, A., Bany Mohammed, A., \& Maqableh, M. (2016). Modeling Factors Affecting Student's Usage Behaviour of E-Learning Systems in Lebanon. International Journal of Business and Management, 11(2), 299.

Tarhini, A., Mgbemena, C., \& Trab, MSA. (2015). User Adoption of Online Banking in Nigeria: A Qualitative Study. Journal of Internet Banking and Commerce, 20(3), 1-8.

Tiwari, R. (2008). Defining Innovation, Hamburg University of Technology, Research Project Global Innovation, 1-3.

Ungerman, O., Dedkova, J. \& Gurinova, K. (2018). The Impact of Marketing Innovation on The Competitiveness Of Enterprises in The Context of Industry 4.0. Journal Of Competitiveness, 10(2), 132-148.

Yassien, E., \& Mufleh, M. (2017). The Impact of ERP System's Usability on Enterprise Resource Planning Project Implementation Success via the Mediating Role of User Satisfaction. Journal of Management Research, 9(3), 49-71.

Yeh, T.M., Chen, S.H., \& Chen, T.F. (2019). The Relationships among Experiential Marketing, Service Innovation, and Customer Satisfaction-A Case Study of Tourism Factories in Taiwan. Sustainability, 11(4), 1041 
Zheng, Y., Wang, J., Tsai, S.-B., Li, G., Wang, J., \& Zhou, J. (2017). Research on Customer Satisfaction in Marine Cultural and Sustainable Tourism-A Case Study of Shanghai.

Sustainability, 9, 1-11.

Zuñiga-Collazos, A., \& Castillo-Palacio, M. (2016). Impact of Image and Satisfaction on Marketing Innovation. Journal of Technology Management \& Innovation, 11(2), 70-75. 